\title{
Polarization signals in the marine environment
}

\section{Thomas W. Cronin, Nadav Shashar, Roy L. Caldwell, Justin Marshall, Alexander G. Cheroske, et al.}

Thomas W. Cronin, Nadav Shashar, Roy L. Caldwell, Justin Marshall, Alexander G. Cheroske, Tsyr-Huei Chiou, "Polarization signals in the marine environment," Proc. SPIE 5158, Polarization Science and Remote Sensing, (12 December 2003); doi: 10.1117/12.507903

SPIE Event: Optical Science and Technology, SPIE's 48th Annual Meeting, 2003, San Diego, California, United States 


\title{
Polarization signals in the marine environment
}

\author{
Thomas W. Cronin ${ }^{* a}$, Nadav Shashar ${ }^{\mathrm{b}}$, Roy L. Caldwell ${ }^{\mathrm{c}}$, Justin Marshall ${ }^{\mathrm{d}}$, Alexander G. Cheroske ${ }^{\mathrm{a}}$, \\ Tsyr - Huei Chiou ${ }^{a}$ \\ ${ }^{\mathrm{a}}$ Dept. of Biological Sciences, University of Maryland Baltimore County, Baltimore, MD, \\ USA 21250 \\ ${ }^{\mathrm{b}}$ Interuniversity Institute of Eilat, P.O. Box 469, Eilat, Israel 88103 \\ ${ }^{c}$ Dept. of Integrative Biology, University of California, Berkeley, CA, USA 94720 \\ ${ }^{\mathrm{d}}$ Vision, Touch, and Hearing Research Centre, University of Queensland, Brisbane, Queensland \\ 4072, Australia
}

\begin{abstract}
Although natural light sources produce depolarized light, partially linearly polarized light is naturally abundant in the scenes animals view, being produced by scattering in air or water or by reflection from shiny surfaces. Many species of animals are sensitive to light's polarization, and use this sensitivity to orient themselves using polarization patterns in the atmosphere or underwater. A few animal species have been shown to take this polarization sensitivity to another level of sophistication, seeing the world as a polarization image, analogous to the color images humans and other animals view. This sensory capacity has been incorporated into biological signals by a smaller assortment of species, who use patterns of polarization on their bodies to communicate with conspecific animals. In other words, they use polarization patterns for tasks similar to those for which other animals use biologically produced color patterns. Polarization signals are particularly useful in marine environments, where the spectrum of incident light is variable and unpredictable. Here, cephalopod mollusks (octopuses, squids, and cuttlefish) and stomatopod crustaceans (mantis shrimps) have developed striking patterns of polarization used in communication.
\end{abstract}

Keywords: polarized light, polarization sensitivity, polarization vision, biological signal, marine animal

\section{INTRODUCTION}

Many animals besides humans use visual signals for communication, both within a species (intraspecifically) and between species (interspecifically). To be effective, such signals should be highly visible to intended receivers, but often should be inconspicuous or invisible to unintended ones. Animals may control the visibility of their signals by making them highly directional, by displaying them only transiently or under only the most appropriate lighting conditions, or by producing colors that are poorly detected by undesirable receivers ${ }^{1}$. Only recently have we become aware of a special class of visual signals that are highly visible to some animals, including the intended receivers, but undetectable to others, including vertebrate predators. These signals are based on the controlled reflection of partially linearly polarized light, using specialized biological effectors to produce the desired reflection.

\subsection{Polarized light in natural environments}

Light arriving from the sun and moon is fully depolarized, consisting of mixtures of photons having randomly oriented $e$ vectors. Nevertheless, partially linearly polarized light is found throughout most natural scenes, as it is readily produced by scattering in the atmosphere or under water, or by reflection from natural surfaces such as the air/water interface and the shiny cuticles of leaves or arthropods (particularly insects and crustaceans). These natural patterns of polarization are readily visualized by imaging polarimeters ${ }^{2,3}$, and the patterns themselves are often predictable (though changeable throughout the day) and thus usable for animal orientation ${ }^{4}$, particularly in air. Under water, especially away from the surface, polarization patterns are generally simpler than those in the air, because refraction at the air/water interface causes light from the sky or other celestial sources to be confined to a cone of light about $97^{\circ}$ across. When this downwardly oriented light is scattered in water, the predominant angle of polarization ( $e$-vector angle) is generally near the horizontal plane. However, the degree of polarization under water is normally quite low, rarely reaching $70 \%$ and

*cronin@umbc.edu; phone 1410 455-3449; fax 1410 455-3875 
and generally being much less ${ }^{5,6}$. Also, polarized reflection of light from underwater objects is not generally favored, since the refractive index of water greatly exceeds that of air, reducing the specular reflection of light from surfaces. Thus, polarization throughout an underwater scene is almost always low (Fig. 1). This actually favors polarization signaling in water. The pattern of polarization is simple, so local deviations in polarization should be easily detectable, and the degree of polarization is low, making well-polarized signals stand out. Although polarization signaling is known to exist in insects ${ }^{7}$, and could be used by other terrestrial animals, it is most highly developed in marine invertebrates.

Figure 1: Distribution of polarized light in an underwater scene. This photograph illustrates a scene on the Great Barrier Reef, Australia in clear water at a depth of about $20 \mathrm{~m}$, near midday. Panel A shows the reef as a standard black-and-white photograph. The image of Panel B was obtained by taking 3 digital photographs of the scene, through a linear polarizer rotated to 0,45 , and 90 degrees successively and then analyzing them pixel-by-pixel according to Wolff and Andreiou $^{2}$ to show the degree of polarization (here, as $\%$ polarization) throughout the scene. The inset in Panel B shows the scale into which polarization content is coded, where white represents $100 \%$ polarized light. Note that the degree of polarization is low everywhere in the scene, despite the clear water and the bright overhead lighting.

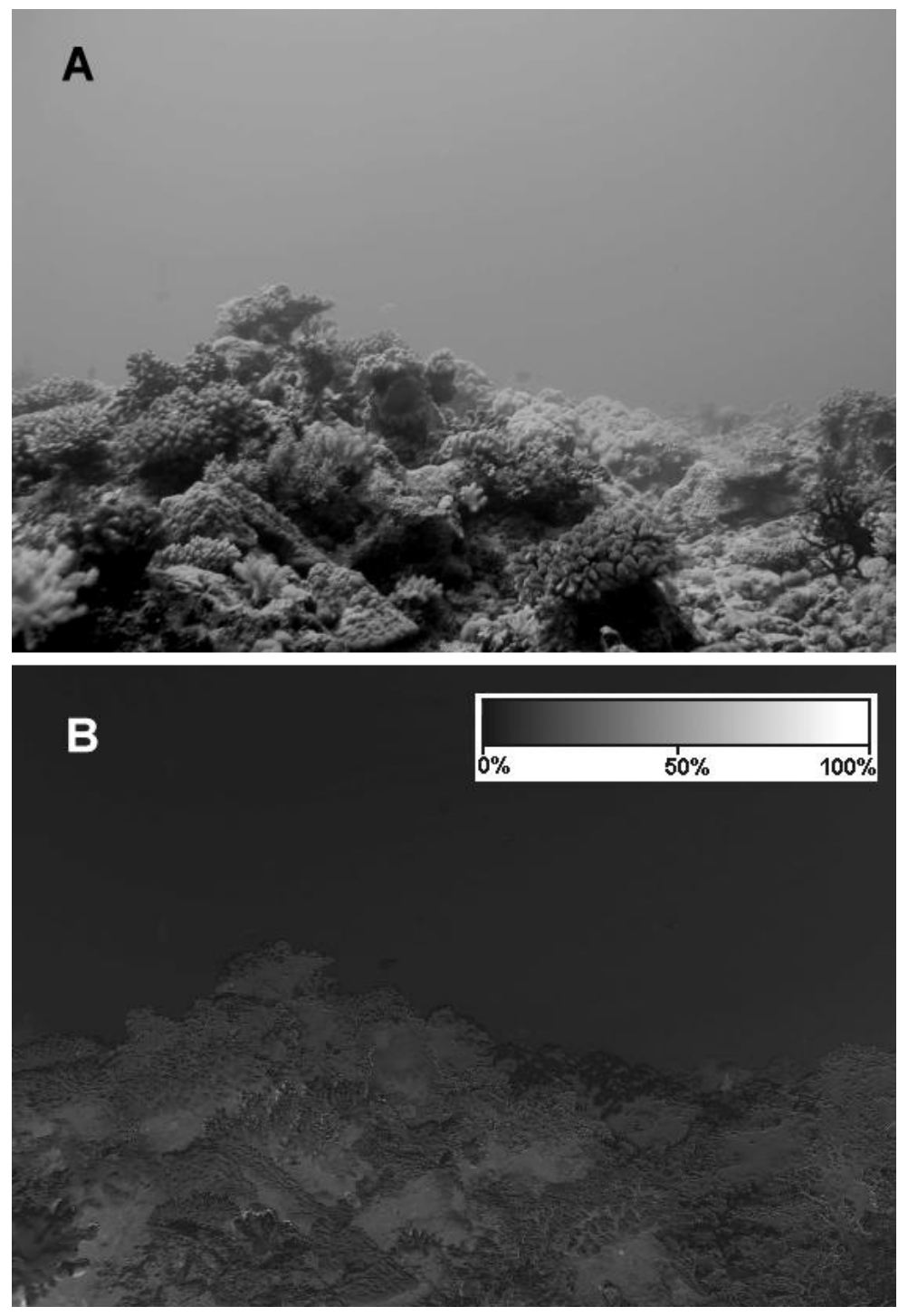

\subsection{Polarization vision and biological uses of polarized light}

Because humans are essentially insensitive to the polarization properties of light, it is easy to overlook the fact that most photoreceptors are inherently capable of responding differentially to the $e$-vector orientation of linearly polarized light. This occurs because visual pigment molecules, the chemicals that actually capture light and begin the process of phototransduction, are dichroic. Each chromophore, the region of the molecule that absorbs light of visible wavelengths, absorbs a photon most effectively when its $e$-vector is aligned parallel to the chromophore's axis. Molecules of visual pigment are confined to the membranes of photoreceptor cells. Their absorption dipoles tend to be oriented more or less parallel to the membrane's surface, but may take on any angle in the plane of the membrane. Since the disks or lamellae 
of vertebrate rods and cones are oriented with their surfaces perpendicular to the path of incoming light, the visual pigments they contain are deployed at random orientations to the light, and the receptor as a whole is normally insensitive to polarization (i.e. it responds equally to all planes of polarization). This is true in principle for all vertebrate photoreceptors, yet there are some vertebrate species that respond to polarized light using poorly understood receptor mechanisms ${ }^{8,9}$.

Photoreceptors of most invertebrate species are constructed differently from those of vertebrates. Visual pigments are localized in membranes of groups of microvilli, and the photoreceptive region of the receptor cell looks much like a stack of pencils, all oriented in parallel. Again, chromophores of visual pigments lie parallel to the plane of the membrane, but since the membranes are curved into tiny cylinders, the pigment molecules tend not to rotate within the membrane, but instead to orient with their absorption dipoles roughly parallel to the axis of each microvillus. Since the most common situation is for all microvilli in a cell to be parallel, the entire cell responds much more strongly to light when the $e$-vector of polarization is parallel to the microvillar axes than when it is otherwise oriented. Many invertebrates take advantage of this inherent polarization sensitivity to analyze polarized light stimuli. Most commonly, there are two polarization classes of receptors, each class having microvilli parallel throughout the retina and orthogonal to the microvilli of the opposite class. Thus, the polarization system relies on 2-axis analysis, although eye or body movements (or an additional class of polarization-insensitive receptors) can provide additional information to permit the full analysis of partially linearly polarized light, extracting the intensity, degree of polarization, and $e$-vector angle.

Polarization sensitivity in arthropods has long been associated with orientation to celestial patterns of polarization ${ }^{4}$, and some fishes may use polarization in orientation behavior as well ${ }^{8}$. This type of behavior does not require good imaging capability to see the polarization pattern, and may even use a specialized subset of photoreceptors devoted to these tasks. Some animals, however, actually see patterns of polarized light, a visual experience analogous to color vision (where the scene is segregated into regions based on different spectral radiances). Thus, by analogy, this ability is called polarization vision, and it is found in octopus and other cephalopods ${ }^{10,11}$, as well as in mantis shrimps ${ }^{12}$. It can be useful for detecting transparent or well-camouflaged animals in the low contrast underwater environment $t^{1,13,14}$.

\section{POLARIZATION SIGNALS IN MARINE ANIMALS}

\subsection{Polarization signals in cephalopod molluses}

Once an animal has evolved polarization vision, it is easy to see how polarization signaling could then appear. Cephalopods (octopuses, squids, and cuttlefish) are well-known for their sensitivity to polarized light. Nevertheless, the discovery in cuttlefish of polarized-light patterns that could quickly be switched on and off was unexpected ${ }^{15}$. Similar patterns were subsequently found in octopuses and squids ${ }^{16,17}$ (Fig. 2). The polarization is produced by reflection from oriented stacks of platelets, probably made of guanine, found in specialized cells called iridophores. These cells are

Figure 2: Reflection of polarized light from the squid, Loligo pealei. Panel A shows a standard black-and-white image of the anterior region of the animal, showing mainly the arms of the squid. Panel B represents orientation of polarization ( $e$-vector angle), and $\mathrm{C}$ represents the degree of polarization ( $\%$ polarization); see the insets for figure interpretation. Note that the arms of the squid reflect light of two different orientations of polarization (arrows). Images were obtained using a Lowe-type polarization camera ${ }^{18}$.
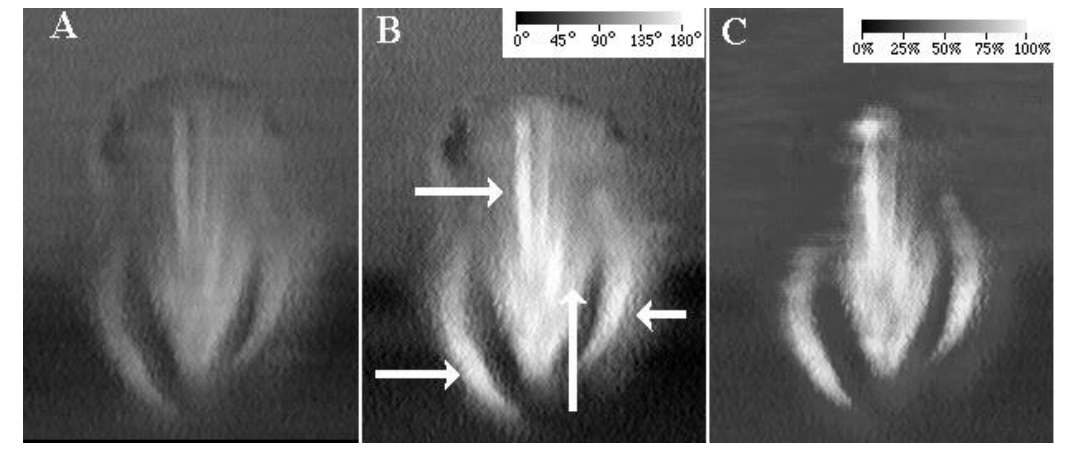

located just under the surface of the skin, and they are actually a type of cellular effector, capable of changing their internal organization under nervous control ${ }^{16,19}$. Therefore, an individual squid or cuttlefish can "turn on" its polarization pattern at will, or alternatively, "switch it off", in less than a second. Videotapes of these animals reveal a dynamic shifting of patterns, but at present, we still do not know what specific information is carried in these signals. Research 
has shown that they appear in particular behavioral contexts ${ }^{16,20}$, but how they may affect behavior in the presumed target individual, or even whether they carry any specific meaning at all, remains unknown.

\subsection{Polarization signals in stomatopod crustaceans}

Stomatopod crustaceans, also known as mantis shrimps, are violent marine predators that roam coral reefs or hunt from burrows, taking prey by bashing or stabbing it with a modified raptorial appendage. They have unique and extremely specialized compound eyes with as many as 16 spectral channels (ranging from the deep ultraviolet to the far red) ${ }^{21}$, as well as a receptor subset whose anatomy suggests a high degree of structural specialization for the analysis of polarized light ${ }^{21,22}$. In addition, they are the only crustaceans definitively proven to be capable of color vision ${ }^{23}$, and the only ones demonstrated to have polarization vision ${ }^{12}$. Mantis shrimps are often beautifully colored, and their color patterns and spots are important in intraspecific and interspecific signaling ${ }^{24}$. Recently, it has been found that some species, all of which have diurnal activity patterns, use polarization signals as well. The parts of the body that produce the polarized light reflections are the same structures that, in other species, are brightly colored and clearly involved in signaling. These include the maxillipeds (feeding appendages displayed under the "head"), the antennal scales (flap-like appendages extending laterally from the region of the eyes), parts of the carapace itself, and the uropod scales ("tail fan" $)^{25}$. The particular regions used vary among species, and a given individual can have several different parts, or only one, involved in polarization signaling. Here, we will discuss polarized-light signals in stomatopods with reference to two species in the genus Haptosquilla. These make an interesting comparison, because while closely related and often found living in close proximity to each other, the polarization produced by each species appears on a different body part, and the spectral properties of the reflectances are so different in the two species that they imply rather different optical systems underlying the production of the polarization. Both species described here were collected at the Lizard Island Research Station, on the Great Barrier Reef in Australia.

\subsubsection{Haptosquilla trispinosa}

H. trispinosa is found living over a large range of depths, from just subtidally (depth $<0.5 \mathrm{~m}$ ) to greater than 30 meters deep. Consequently, the spectral properties of the illumination within which its signals are viewed are somewhat variable, which may have encouraged the evolution of polarization signals. The latter are more constant in appearance, since they do not depend strongly on the spectrum of the illuminant. H. trispinosa is a small species, living in chambers in coral rubble or rock, from which it hunts. Figure 3 shows an individual in its burrow, where it is able to survey the surroundings with its excellent eyes as well as its extended antennules, and from which it can easily signal to passing individuals. The polarizing region is found on the first maxilliped, an appendage associated with feeding and with cleaning behavior in the stomatopods, indicated by an arrow in Fig. 3A. It appears bright, shiny blue in color and is quite visible from outside the burrow. Views of a dissected segment, where the polarizer is located, are provided in Fig. $3 \mathrm{~B}$, illustrating how the reflectance is strongly horizontally polarized. Much of the reflection is excluded when viewed through a vertical analyzer (Fig. 3B, bottom half).

Spectral properties of the polarization were examined using an Ocean Optics USB2000 spectrometer attached to a fiber optic mounted to a camera on a compound microscope. With this arrangement, polarization from a spot less than a millimeter in size can be measured. Three spectra are collected, each referenced to a LabSphere Spectralon diffuse reflection standard, at $0^{\circ}, 45^{\circ}$, and $90^{\circ}$. From these, the average reflectance, degree of polarization, and $e$-vector angle can be computed at each wavelength ${ }^{2}$. Results of this analysis are plotted in Fig. 3C. Note that the polarization is horizontal (near $180^{\circ}$ ) throughout the spectrum, as suggested by the images in Fig. 3B. Reflectance peaks sharply at about $475 \mathrm{~nm}$, but the peak of polarization extends from about $460 \mathrm{~nm}$ to near $550 \mathrm{~nm}$, where the degree of polarization is consistently near $70 \%$. Receptors of $H$. trispinosa thought to be principally responsible for the analysis of polarized light have peak sensitivity over precisely this spectral range ${ }^{26}$, further supporting the view that this is a tuned signaling system. As of yet, the structure producing the polarized-light signal is not known. Structural and ultrastructural examination of the material is planned. The blue-reflecting region appears to be a system of parallel fibers, oriented perpendicular to the primary axis of the favored polarization reflection (in other words, running vertically in Fig. 3B), so we hypothesize that the fibers contain internal structure across the fiber axis that reflects the horizontally polarized light. Individuals of $H$. trispinosa display their polarization signals not only at the burrow entrance, but also when wandering on the coral reef in search of mates. Females remain within the burrow, showing their blue patches, and inspect passersby. Males, when encountering a female within her burrow, will display their first maxillipeds, including the 
polarizer, and follow this with other displays that include motion and possibly chemical stimuli. If the female is receptive, mating takes place at the burrow entrance. We therefore hypothesize that the polarizing signal is used for species and sexual recognition, and possibly also as a threat display directed at potential intruders. There is no obvious difference in the polarizers of males and females in this species.
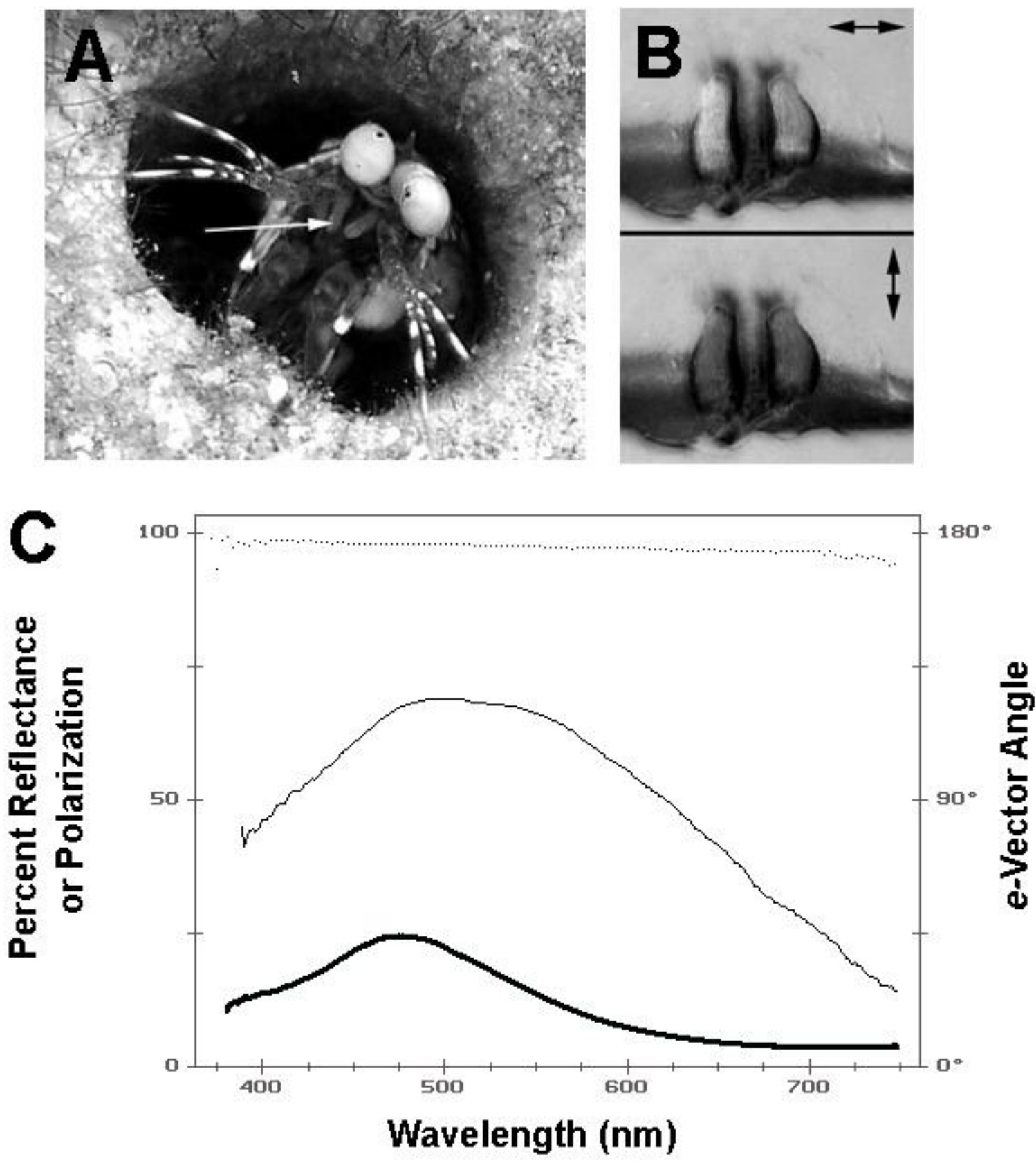

Figure 3: Polarization signals in Haptosquilla trispinosa.. A. An individual of H. trispinosa at its burrow entrance. The white arrow indicates the polarizer on the first maxilliped. The burrow is about $1 \mathrm{~cm}$ across at the entrance. B. Two images of pair of polarizers after dissection from an individual, oriented as they would normally be in life (dorsal to the top). The images were taken through a polarizing analyzer oriented horizontally in the top frame and vertically in the bottom frame, as indicated by the 2-headed arrows. Note the much stronger reflectance when viewed in horizontally polarized light. C. Spectral properties of the polarization. The THICK curve indicates average percent reflectance, showing a peak in the blue (near $475 \mathrm{~nm}$ ). The THIN curve shows the degree of polarization, which reaches about $70 \%$ in the spectral region from $\sim 475 \mathrm{~nm}$ to $\sim 550 \mathrm{~nm}$. The DOTTED curve shows $e$-vector angle, with $0^{\circ}$ and $180^{\circ}$ being horizontal and $90^{\circ}$ being vertical. 


\subsubsection{Haptosquilla glyptocercus}

This species is often collected in exactly the same habitat as H. trispinosa, living side-by-side with its congener. However, in general it tends to be encountered in somewhat shallower waters (intertidally or barely subtidally) and has a much broader geographical range than does $H$. trispinosa. It is also distinctly more polymorphic in coloration, varying from brown to green and even black. In all color morphs, there is always a bright pink or red line across the dorsal abdomen at the joint between the last and next-to-last abdominal segments (Fig. 4A). This strip of color reflects polarized light (Fig. 4B, C). In contrast to H. trispinosa, there is no polarization patch on any of the maxillipeds.
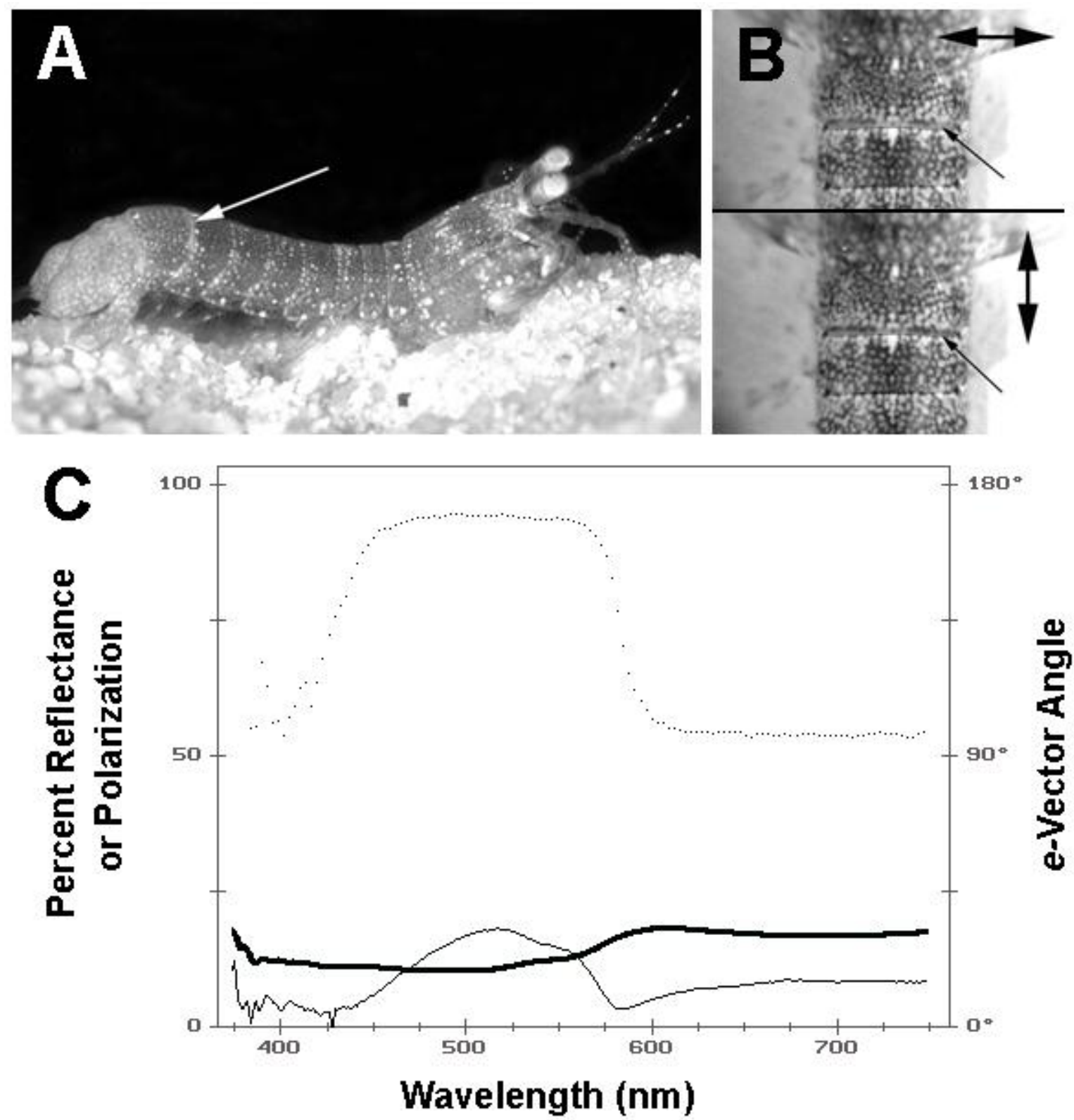

Figure 4: Polarization signals in Haptosquilla glyptocercus. A. An individual of $H$. glytocercus, to show the polarizer at the border between the last two abdominal segments (white arrow). B. Two images of the abdomen of an individual, oriented with posterior to the top. As in Fig. 3, the images were taken through a polarizing analyzer oriented horizontally in the top frame and vertically in the bottom frame, indicated by the 2-headed arrows. Note that the strip between the segments (thin arrows) is somewhat brighter when viewed in horizontally polarized light. C. Spectral properties of the polarization. Curves as in Fig. 3. See text for further discussion. 
Light reflected from the polarizing material in this species has a much lower degree of polarization (Fig. 4C, thin line) than other types of polarizers in stomatopods, suggesting that here the color or contrast of the marking may be more important than its polarization. The polarizer has the unusual property of reflecting light with orthogonal $e$-vector orientation in different spectral regions. Light between $\sim 450 \mathrm{~nm}$ and $\sim 575 \mathrm{~nm}$ is polarized horizontally (e-vector angle near $180^{\circ}$ ), but light in the spectral range beyond $600 \mathrm{~nm}$ is vertically polarized (e-vector angle near $90^{\circ}$ ). The data in Fig. 4 were actually collected from an exoskeleton molted by a newly -collected animal, but data from living animals are similar. Red-colored polarizers are found in several other stomatopod species, and all have the same odd pattern of spectral reflectance ${ }^{25}$, with horizontal polarization at middle wavelengths and vertical polarization at long wavelengths, and a sharp crossover near $575 \mathrm{~nm}$. In these, however, the degree of polarization is much greater, often well above $50 \%$ near $500 \mathrm{~nm}$. In some of these species, the polarization is clearly associated with signaling behavior ${ }^{25}$. We hypothesize that either the color or the polarization, or both, can be involved in signaling when this material is used. Perhaps the polarizer originated in deep-living species, and when these species extended their distribution, or moved into shallower waters where color made a more effective signaling system, the color of the polarizer was retained, but the polarization properties degenerated over evolutionary time as they lost their value. If this is the case, it implies that the polarization requires a well-organized microstructure, which is retained in deep-living species but lost in their shallower relatives. The fact that the polarization is retained in the molt implies that all the required structure exists in the cuticle of the animal. (In contrast, if the cuticle is removed from the "blue" polarizer of $H$. trispinosa, all polarization is lost, and the blue fibers are seen to lie in the region just below the cuticle itself.) The polarizer actually lies in the flexible, arthrodial membrane joining the segments and is most visible when the animal is flexed to extend this membrane.

The biological significance of this red line is also not clear, although it appears in all color morphs of $H$. glyptocercus. It is most prominent in egg-bearing females, partly because the abdomen is distended at this time, revealing more of the arthrodial membrane. It is possible that the color serves as a sex-recognition signal at this time, but otherwise its function remains to be determined. Unlike $H$. trispinosa, H. glyptocercus spends time hunting outside the burrow, so the red line would be often visible to conspecifics. In other stomatopods with "red" polarizers, the material is associated with mating displays and with burrow defense ${ }^{25}$, behavior much like that with which the "blue" polarizer of $H$. trispinosa is used. Note that the spectral region used for polarization signaling in both polarizer types is the middle-wavelength range, near $500 \mathrm{~nm}$, the spectral location of polarization-specialized photoreceptors in stomatopods ${ }^{22,26,27}$.

\section{SUMMARY AND CONCLUSIONS}

Biological signals that use color, pattern, brightness, and motion are well known. Here, we discuss a new class of visual signals based on the reflection of polarized light. These signals are particularly useful in the marine environment, specifically in deeper water, where they are favored by the relatively low degree of polarization in the background or in light reflected by most other objects, and by the predictable direction of lighting from overhead. Also, spectral features of illuminating light opt against the use of color signals: the light is either spectrally narrow (nearly monochromatic in deep water) or is spectrally variable as depth, water quality, or viewing distance change. Polarization signals, particularly those designed for use in the wavelength range best transmitted in natural waters (450 to $550 \mathrm{~nm}$ ), are much more resistant to these effects. The structural and optical properties of the materials that produce the polarization patterns are fairly well understood in cephalopod mollusks, but in the stomatopod crustaceans the means by which the polarization is produced require further examination. Polarization signals have the desirable property of being very visible to conspecifics or other intended target organisms, but cryptic or nearly invisible to unintended receivers.

\section{ACKNOWLEDGEMENTS}

We thank the staff of the Lizard Island Research Station for support and assistance. This work is based on research supported by the National Science Foundation under Grant Number IBN-0118793, by the Binational Science Foundation under Grant Number 1999040, and by the Air Force Office of Scientific Research under Grant Number 02NL253.

\section{REFERENCES}

1. J. Endler, "Evolutionary implications of the interaction between animal signals and the environment", Animal Signals, Y. Espmark, T. Amundsen, and G. Rosenqvist (eds), pp. 11-46, Tapir Academic Press, Trondheim Norway, 2000. 
2. L.B. Wolff and A.G. Andreiou, "Polarization camera sensors", Image and Vision Computing, 13, 497-510, 1995.

3. N. Shashar, T.W. Cronin, G. Johnson, and L.B. Wolff, "Portable imaging polarized light analyzer", SPIE, 2426, 30$35,1995$.

4. R. Wehner, " Polarization vision - a uniform sensory capacity?", Journal of Experimental Biology, 204, 2589-2596, 2001.

5. T.W. Cronin and N. Shashar, "The linearly polarized light field in clear, tropical marine waters: spatial and temporal variation of light intensity, degree of polarization, and e-vector angle", Journal of Experimental Biology, 204, 2461-2467, 2001.

6. I. Novales Flamarique and C.W. Hawryshyn, "Is the use of underwater polarized light by fishes restricted to crepuscular time periods?", Vision Research 37, 975-989, 1997.

7. A. Sweeney, C. Jiggins, and S. Johnsen, "Polarized light as a mating signal in a butterfly", Nature, 423, 31-32, 2003.

8. C.W. Hawryshyn, "Polarization vision in fish", American Scientist, 80, 164-175, 1992.

9. J.B. Phillips and J.A. Valdvogel, "Celestial polarized light patterns as a calibration reference for sun compass of homing pigeons", Journal of Theoretical Biology, 131, 55-67, 1988.

10. N. Shashar and T.W. Cronin, "Polarization contrast vision in Octopus", Journal of Experimental Biology, 199, 9991004, 1996.

11. N. Shashar, R.T. Hanlon, and A.M. Petz, "Polarization vision helps detect transparent prey", Nature, 393, 222-223, 1998.

12. N.J. Marshall, T.W. Cronin, and N. Shashar, "Behavioral evidence for polarization vision in stomatopods reveals a potential channel for communication", Current Biology, 9, 755-758, 1999.

13. J.N. Lythgoe and C.C. Hemmings, "Polarized light and underwater vision", Nature, 213, 893-894, 1967.

14. N. Shashar, R. Hagan, J.G. Boal, and R.T. Hanlon, "Cuttlefish use polarization sensitivity in predation on silvery fish", Vision Research, 40, 71-75, 2000.

15. N. Shashar, P. Rutledge, and T.W. Cronin, "Polarization vision in cuttlefish: a concealed communication channel?", Journal of Experimental Biology, 199, 2077-2084, 1996.

16. N. Shashar and R.T. Hanlon, "Squids (Loligo pealii and Euprymna scolopes) can exhibit polarized light patterns produced by their skin", Biological Bulletin, 193, 207-208, 1997.

17. L.M. Mäthger and E.J. Denton, "Reflective properties of iridophores and fluorescent 'eyespots' in the loliginid squid Alloteuthis subulata and Loligo vulgaris", Journal of Experimental Biology, 204, 2103-2118, 2001.

18. R.T. Hanlon, M.R. Maxwell, N. Shashar, E.R. Loew, and K.L. Boyle, "An ethogram of body patterning behavior in the biomedically and commercially valuable squid Loligo pealei off Cape Cod, Massachusetts", Biological Bulletin, 197, 49-62, 1999.

19. K.M. Cooper and R.T. Hanlon, "Correlation of iridescence with changes in iridophore platelet ultrastructure in the squid, Lolloguncula brevis", Journal of Experimental Biology, 121, 451-455, 1986.

20. N. Shashar, C.A. Milbury, and R.T. Hanlon, "Polarization vision in cephalopods: neuroanatomical and behavioral features that illustrate aspects of form and function", Marine and Freshwater Behavior and Physiology, 35, 5768, 2002.

21. T.W. Cronin and N.J. Marshall, "The visual world of mantis shrimps", Complex Worlds From Simpler Nervous Systems, F.P. Prete (ed.), MIT Press, Boston, in press.

22. N.J. Marshall, M.F. Land, C.A. King, and T.W. Cronin, "The compound eyes of mantis shrimps (Crustacea, Hoplocarida, Stomatopoda). I. Compound eye structure: The detection of polarized light", Philosophical Transactions of the Royal Society Series B 334, 33-56, 1991.

23. N.J. Marshall, J.P. Jones, and T.W. Cronin, "Behavioural evidence for color vision in stomatopod crustaceans", Journal of Comparative Physiology A, 179, 473-481, 1996.

24. R.L. Caldwell and H. Dingle, "Ecology and evolution of agonistic behavior in stomatopods", Naturwissenschaften 62, 214-222, 1975.

25. T.W. Cronin, N. Shashar, R.L. Caldwell, J. Marshall, A.G. Cheroske, and Tsyr-Huei Chiou, "Polarization vision and its role in biological signaling", Integrative and Comparative Biology, in press.

26. T.W. Cronin, R.L. Caldwell, and M. Erdmann, "Tuning of photoreceptor function in mantis shrimp species occupying a range of depths. I. Visual pigments", Journal of Comparative Physiology A, 188, 179-86, 2002.

27. T.W. Cronin, N.J. Marshall, and R.L. Caldwell, "Spectral tuning and the visual ecology of mantis shrimps", Philosophical Transactions of the Royal Society Series B, 355, 1263-1267, 2000. 